\title{
Exploring the Way forward for Chinese Creative Design from the Viewpoint of Cybernetics
}

\author{
Qianyu Wu \\ School of Fine Arts, Nanjing Normal University, Nanjing 210023, China. \\ wuqianyu_wqy@163.com
}

\begin{abstract}
This paper discusses some current problems of creative design in China, and introduces the development of cybernetics and its influence on other disciplines. Based on the method of analogy and guided by cybernetics, this work studies the corresponding relationship between cybernetics and creative design, and puts forward three constructive suggestions for Chinese local creative design from three aspects, including the overall mainstream thinking, the specific implementation level and the ultimate goal.
\end{abstract}

Keywords: creative design, China, cybernetics, analogy.

\section{Introduction}

The term 'creativity' began in Christianity, and believers believed that only God could create it. And creative design ideas now can be said to be the legacy of the Western Renaissance, is based on the product of Western individualism [1]. Broadly speaking, creative design is a kind of material or spiritual wealth that human beings need by understanding nature and grasping the laws of nature to transform nature. On January 22, 2014, Premier Li Keqiang chaired a State Council executive meeting to propose the orientation and measures of the new government on the development of creative design industry. The meeting pointed out that cultural creativity and design services are characterized by high knowledge, high value-added, low consumption and low pollution. The development of local design industry has a direct impact on the magnificent turning from 'Made in China' to 'Created in China', and the process of economic restructuring and upgrading of manufacturing industry in China. In the process of the development of creative design, many design methods, such as brainstorming, association, divergent thinking and inspiration, are summarized through constant practice, discussion and exploration, which open the door to creative design $[2,3]$.

In order to understand the frontier and hotspot research of creative design at local and abroad, this paper inquired 'Creative Design' through Web of Science website, and analyzed from three aspects: age, industry and organization. The following results are obtained, as shown in Figs. 1-4.

As can be seen from Fig. 1, since the end of last century, creative design industry began to gradually enter the field of vision of researchers; in the last decade, this direction has been increasingly prosperous and developed; especially in the last three years, the rapid growth further shows that creative design has been fully valued and widely used in all walks of life. The analysis of Fig. 2 shows that creative design industry involves engineering, education, computer science, business economics, material science, social science, physics, art, etc. It can be seen that the market, economic and social benefits of creative design have been in-depth and beneficial to our work, life and learning growth in various aspects. Looking at Fig. 3, we can see that the main research institutions of creative design are concentrated in MIT, Delft University, Harvard University, etc. The analysis shows that the prosperity and development of creative design industry is mainly concentrated in the developed countries of Western Europe and North America, which is further confirmed in Fig.4. Fortunately, in recent years, creative design in China has been able to break through prejudices, and integrate the unique culture of all ethnic groups and the history and culture of China over the centuries into modern creative design, and have achieved remarkable results. Especially, Chines Academy of Sciences, the benchmark of the domestic representative industry, is devoted to research and strive hard, then get a position in the international community. 


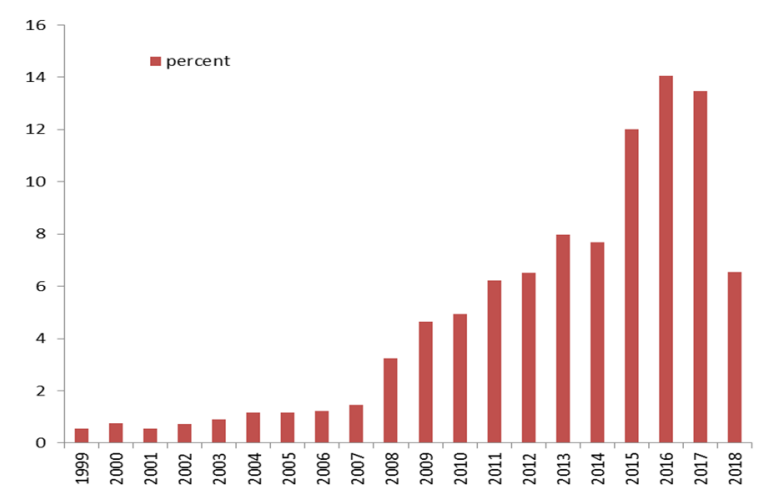

Fig. 1 History of creative design

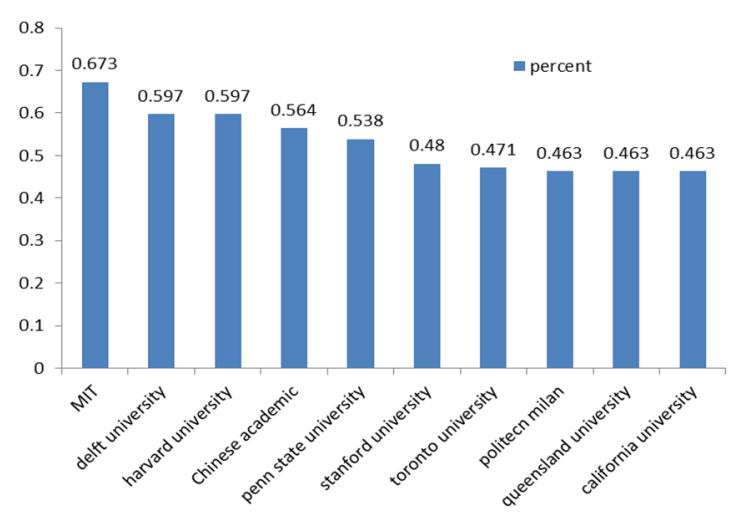

Fig. 3 Organizations in creative design

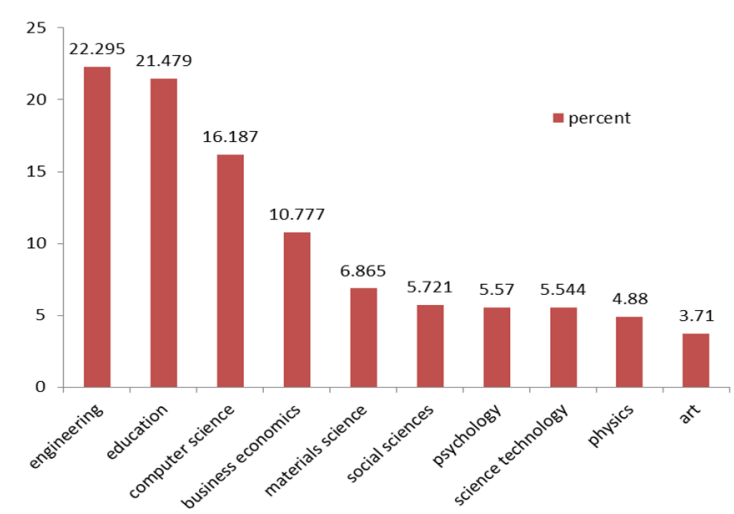

Fig. 2 Subjects in creative design

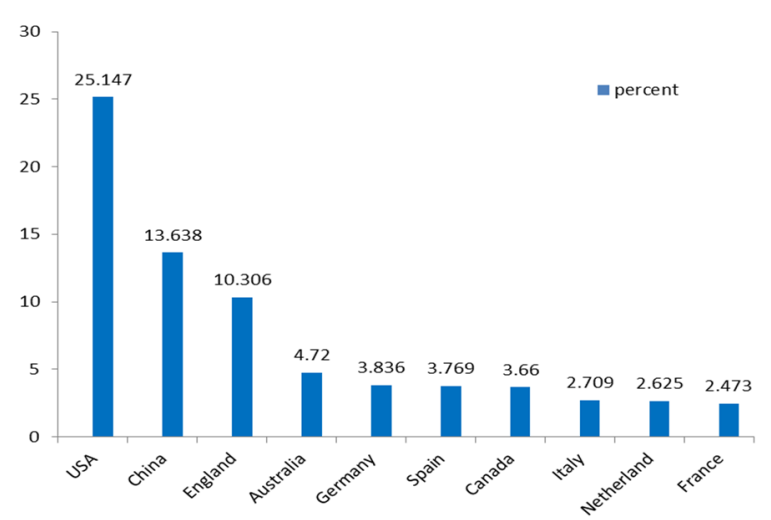

Fig. 4 Districts in creative design

Although China has made great progress in creative design industry, the road of creative design in China has a long way to go. How to explore new ways and new directions is one of the key bottlenecks of current research. Creative design cannot satisfy people's needs simply by satisfying the material life. More creative design with spiritual dimension has become the pursuit of contemporary people, and the human-computer interaction effect in the process of product use has become the customer experience valued by more and more modern consumers [4]. Focusing on the development path of creative design in China, there are still some problems to be discussed.

1) At present, in terms of design ideas, the mainstream of creative design ideas to Western art as the lead, seeking a combination of Chinese and Western ways, the Chinese local creative design has not yet begun. Despite the preliminary exploration of creative design, which combines traditional Chinese culture (folk culture and poetry), it is still confined to the combination within the humanities and social sciences.

2) In terms of specific design, regional culture is characteristic, and the key units (such as the Central Academy of Fine Arts, Academy of Social Sciences, Artists Association, etc.) have not formed a unified platform in China. Although there are preliminary explorations in the form of product pre-release, product crowdsourcing and product design network solicitation, they are still limited to a single product or organization, and cannot share the creative design and maximize the benefits.

3) In terms of propaganda and protection of interests, the system and mechanism are imperfect, piracy and copying are serious (a treasure's clothing style). Despite the brand effect, the domestic independent creative design brand develops slowly and fails to form a good international recognition and status.

This paper adopts the method of analogy, draws lessons from the idea of "bionic" in robot design, the idea of "near human" in artificial intelligence design, the application of Wu opera culture in home product design, the application of Chinese classical poetry image in cultural creative design and other ideological trends, and proceeds from the viewpoint of cybernetics to creative design. The direction of creative design development is discussed in depth. First of all, we briefly introduce the basic 
concepts and development of cybernetics. Secondly, based on the method of analogy, this paper analyzes the common path between cybernetics and creative design. Finally, based on the above analysis, we briefly summarize the future of China's creative design development.

\section{Cybernetics and Development}

Cybernetics is a Greek word originally meant to steer a ship. It contains many meanings, such as regulation, manipulation, management, command, supervision, and so on. In 1943, Wiener and his colleagues published a paper entitled 'The Theories of Behavior, Purpose and Purpose', which is an outline of Wiener's later cybernetics. This paper has two purposes: one is to emphasize the importance of the concept of purpose; the other is to define behaviorism research methods, namely the black box method. In 1948, Weiner published with cybernetics. Until then, it was the first time that different elements of the past were linked together, summarized in a philosophical rather than technical point of view, and a new field was formally born. When Weiner's cybernetics was published, there was a rapid upsurge of communication [5].

Cybernetics is viewed as the discovery of a new continent, marveling at its rich ideological resources and almost unlimited research horizons. It seems that an evolutionary theory has been born with a tidal wave of enthusiasm from academia, the government and the media. Promoted by the joint efforts of the government and the people, cybernetics was rapidly disseminated and applied. It quickly aroused the interest of mathematics, engineering, biology, psychology, political science and other disciplines, and triggered the creation and development of a series of interdisciplinary disciplines in the name of cybernetics, such as biological cybernetics and economic control.

\section{Comparison between Cybernetics and Creative Design}

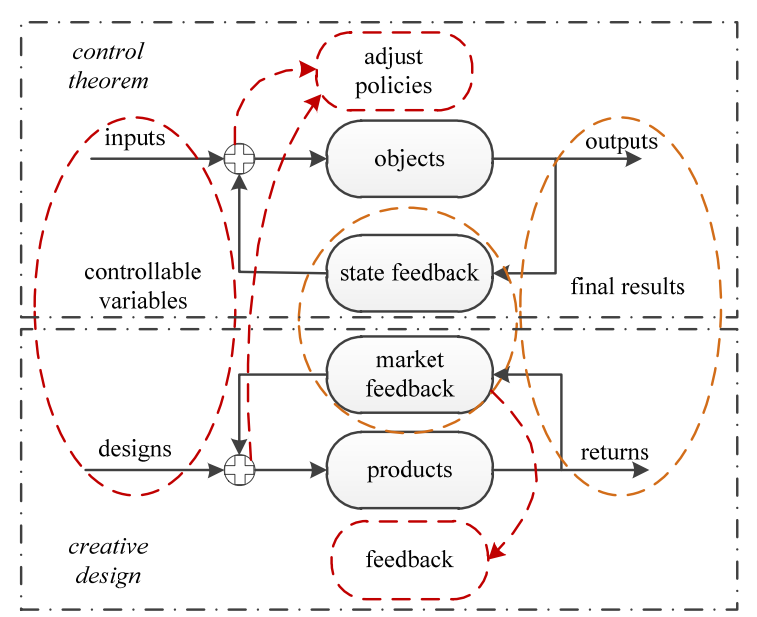

Fig. 5 Relationship between control theorem and creative design

There are three main elements of cybernetics, including control objects, control objectives and control methods. We hope to achieve the desired control performance through a certain control method when expecting a specific control object. The most important thing is what kind of control method is adopted, namely the adjust policies in Fig. 5. It can be seen that in the whole control framework, we are concerned with the control input, final output and state feedback, and can adjust the input control of the control object timely and appropriately to achieve the desired control performance. In contrast, creative design is carried out for a product through the design concept and design methods to get a different product presentation, thus achieving the expected market benefits. Different from cybernetics, the object of creative design is the product, the corresponding input is the market demand, while the final output is advertising, packaging, construction and other products, and the feedback link are market response and customer satisfaction feedback. It is worth learning that the state feedback in the control framework is similar to the market reaction and customer satisfaction 
feedback in creative design. The significance of both lies in the final design strategy adjustment to ensure better and better final results.

\section{Suggestions for Chinese Creative Design based on Cybernetics}

From the perspective of cybernetics, we summarize the detail suggestions as follows.

1) Breaking through traditional concepts, interdisciplinary integration, such as cybernetics, and guiding their own development with the eternal law of the development of natural things, so-called all roads lead to Rome. Interdisciplinary integration has become a major trend of innovation in the current mainstream ideas such as cross-border singers, cross-border comedians, extreme challenges and other variety shows, films and audio-visual types of works. Break trade and regional barriers, fully mobilize the enthusiasm of all sectors of society, promote technological innovation, business form innovation, content innovation, model innovation and management innovation, promote cultural creativity and design services industrialization, specialization, intensive and brand development, promote deep integration with related industries, and bring about new technologies, and process new products to meet new needs.

2) Focusing on the concrete implementation, the forefathers will take the lead one by one, so as to open up new fields and create new directions. Utilizing the advantages of contemporary talent cultivation, we suggest concentrating superior resources such that we can establish and perfect talent cultivation mechanism, and then cultivate innovative talents and establish talent pool. On this basis, the resources and manpower of existing state institutions, relevant enterprises and institutions as well as small and micro start-up companies should be integrated to build a large platform for appreciation of domestic independent creative design and strengthen communication. In this way, we can achieve a fast and efficient market forecasting mechanism and market feedback response mechanism, in order to build a more intimate, more thoughtful and more responsive mechanism for product design in creative design.

3) Ultimately, similar to cybernetics, state tracking and achieving expected performance indicators, on the basis of the first step leading the trend of thought and the second step firmly implemented, the final product will be put into the market in the best time, the best area and the largest audience to achieve precise delivery, thus ensuring the maximum expected market return. Moreover, more commercial rights and interests should be guaranteed for self-built and self-owned brands, so as to ensure the rapid development of self-owned brands and eventually to go out of the country and benefit all over the world.

\section{References}

[1]. Qinqin Ren. Oriental aesthetics and creative design lead Chinese design to the world. Textile Apparel Weekly. (2018) No. 3, p. 32.

[2]. Yuanpu Jin. Three design cities are leading China's creative design into the world. Journal of Ocean University of China (Social Sciences Edition). (2014) No. 5, p. 31-38.

[3]. Yingyi Zhang. Exploring the important role of natural form in creative design. Art and design. Popular literature and art. (2017) No. 8, p. 75.

[4]. Hui Li. Application of interaction design in product creative design. Science and Technology \& Innovation. (2017) No. 4, p. 136, 138.

[5]. Baiwu Wan. Development trend of cybernetics in the 21 st century (review): To commemorate the 70 anniversaries of the cybernetics since founding (1948-2018). Control Theory \& Applications. Vol. 35 (2018) No. 1, p. 1-12. 